\title{
Towards a Systemic Approach to Fire Safety
}

\author{
ALAN N. BEARD \\ Unit of Fire Safety Engineering, University of Edinburgh \\ King's Buildings \\ Edinburgh, EH9 3JL, United Kingdom
}

\section{ABSTRACT}

In order to understand fully the nature of fire safety, which encompasses social values and engineering hardware, it is necessary to consider it as a part of a 'dynamic whole'. Fire may be regarded as a failure of a system. A methodology which may be of help in attempting to approach fire safety from this point of view is suggested.

\section{INTRODUCTION}

Fire safety is often considered in a fragmentary way. That is to say, the elements which combine to produce fire and possible loss of life or injury are often effectively regarded as independent of each other. Such a disjointed approach must inevitably lead to a superficial appreciation of the problems. In order to gain a deep and comprehensive awareness of the nature of the risk in a particular situation it is necessary to attempt to consider all aspects of the problem in a coherent way. As a part of this an elucidation of the factors involved and the pertinent relationships is vital. The fire risk in a given situation is a result of the interaction of a number of 'parts'. That is, fire safety is a characteristic of an entire system and in order to understand fire safety it is necessary to understand the system.

\section{SYSTEMIC APPROACH}

The word 'system' has been used in many different ways and it is suitable here to adopt the broad definition that a system is any entity, conceptual or physical, which consists of inter-dependent 'parts'. The word 'parts' has been put in inverted commas as there is discussion as to just what a 'part' is. (See, for example, ref. 1). However, such considerations will not be pursued further here and it will be assumed that a 'part' may be fairly easily understood. A closed system is such that no interaction takes place with elements outside the system. Ultimately there is only one closed system i.e. the Universe. Smaller systems will be open to a greater or lesser extent.

A system may be considered as 'failed' if there are aspects of the system which are regarded as undesirable by one or more people involved. Whether or not something represents a 'failure' depends upon one's point of view and position within the entire system. With this in mind it is possible to think of fire as a failure of a system. 
In order to gain a full understanding of the fire situation it is necessary to consider the systems involved and to look beyond the immediate horizon. There is a need for a 'systemic approach' to the fire problem and a systemic approach is not the same as a systematic approach. The word 'systematic' may be thought of as implying 'methodical' or 'tidy', but 'systemic' implies something else. A systemic approach is to see the 'dynamic wholeness' in a situation. It is a way of looking at things which should help one to see pattern and inter-relationship within a complex whole. A mode of thinking may be systematic and yet not be systemic. The significance of the concept of the 'whole' has been graphically and simply illustrated by M'Pherson (2). He takes the example of a swarm of gnats as a 'whole' and points to "the fact that each and every gnat turns back towards the centre of the swarm whenever it finds itself at the edge, which is a behavioural property that cannot be understood by only counting the gnats and tracking their motion".

The realization that it is necessary to look upon things as a dynamic whole is not new; it goes back to at least 500 B.C. At about that time Heraclitus put forward his idea that everything is in a state of perpetual change. The 'essence of Being is becoming' and all is part of the 'Universal flux':

"One cannot step into the same river twice, nor touch substance twice in the same state ....

Into the same rivers we step and we do not step."

It is interesting that Heraclitus regarded all bodies as transformations of just one element - fire. If anything, it is more important to have this active, all embracing, view of things today than it was 2,500 years ago. A problem needs to be seen in its context and not in isolation.

In order to carry out a 'systemic study' it is necessary to have an idea of the objectives of the study and an appropriate methodology. Which methodology is suitable depends upon the study objectives and the nature of the systems involved. It is useful to consider systems as either 'hard' or 'soft' (3). A 'hard' system is one in which the parts and relationships are well defined and quantified, such as an engineering system. A 'soft' system is one in which not all the parts and relationships are easily defined and quantified. All systems in which human beings play a large part are essentially 'soft'. Also, it may not be possible to give exact expression to the objectives of a study of a soft system, at least at the beginning.

\section{METHODOLOGY FOR FIRE SAFETY}

A suggested methodology for fire safety, taking into account its systemic setting, is shown in figure 1 .

Although this methodology is written as a series of steps it should not be regarded as a 'sausage machine' which, when completed, produces a 'correct answer'. The need will probably arise to return to earlier steps and cycle round. It will almost certainly be necessary to return to the stage 'Formulation of the problem' more than once. The methodology should be regarded as dynamic and not static.

The 'problem' may become very complex and usually there cannot be a single simple 'solution'. 


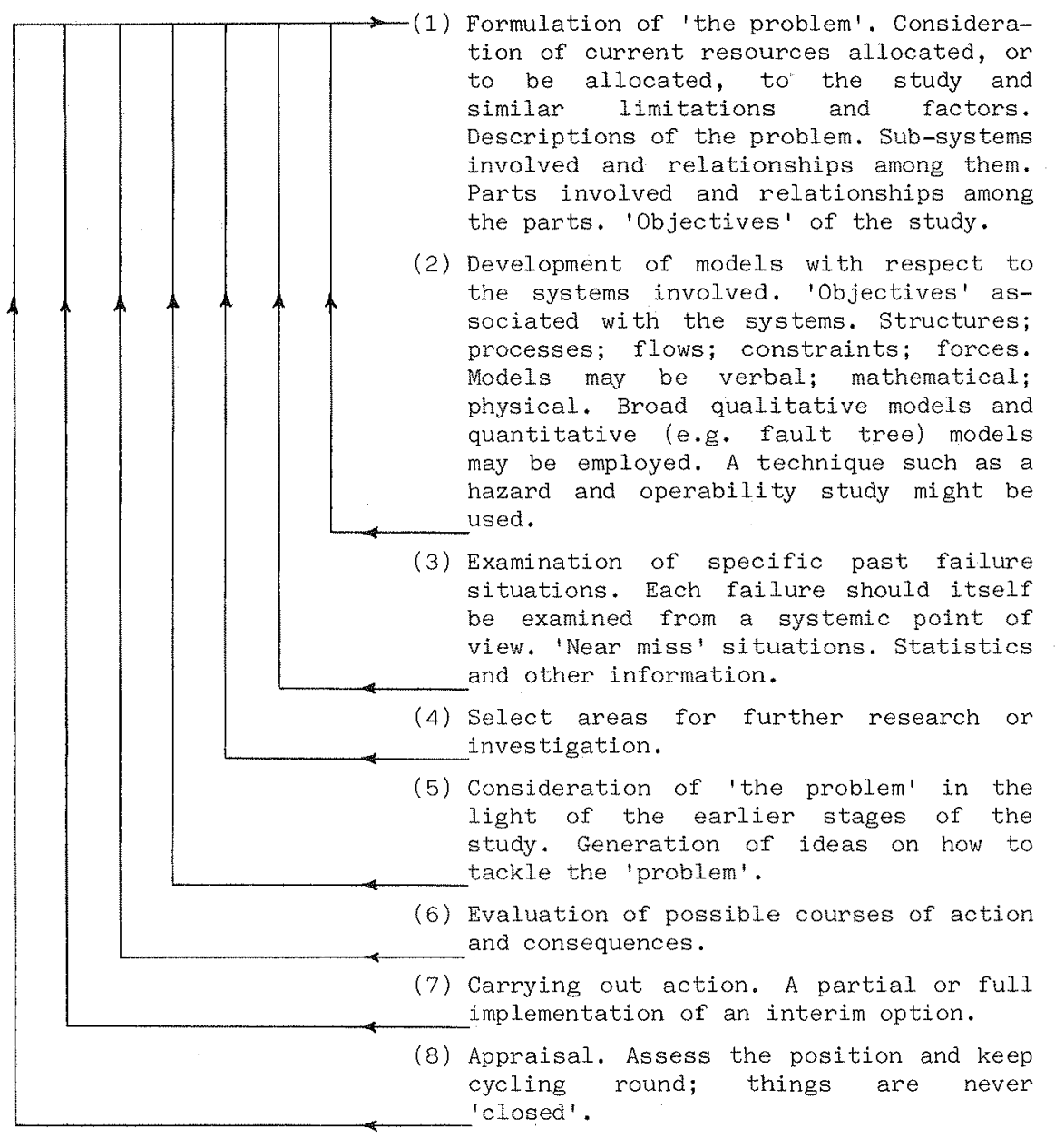

FIGURE 1: A suggested methodology for a systemic study in relation to fire risk.

A problem will, in general, require cycling through the stages of the methodology indefinitely. That is, in principle, a never-ending series of iterations may be required. The statement of 'the problem', and understanding of it, will change through time. Intermediate working solutions may be formulated at different points in this process. Although the difficulties are great it is suggested that the methdology does provide a guiding structure for tackling a situation. Within the structure techniques may be used which may be 'sausage machines' to a greater or lesser degree.

Both theoretical and experimental models might be used. For example, a theoretical model might be constructed which aimed at providing the probability of fatalities given ignition. Arother model might be of a purely verbal kind relating, for example, bed-sores in a hospital ward to the type of mattress or the staffing level. Such knowledge might be put forward by 
nursing staff. Different forms of model should be considered in an attempt to throw light on different facets of the overall system. It must be stressed that a model does not constitute a systemic approach itself. However, models may be used as part of a systemic approach; a model would attempt to answer a relatively limited question within a broader consideration. Within its structure a technique may bring in many different factors. However, a distinction must be drawn between a model which brings in many different factors as part of an attempt to answer a particular question and a systemic approach itself. (More is said about models later in this paper). In short, a systemic approach should not be dogmatic and prescriptive but open and flexible. The tentative methodology outlined here may be thought of as an initial attempt to provide a green field within which people may fruitfully graze rather than an algorithm for the forced feeding of a goose. Some comments on the stages are in order:

(1) Formulation of 'The Problem'. Why is the study being carried out? What has prompted it? What are the initial objectives of the study? In the case of hospitals the 'problem' might be rather tentatively stated as 'How to increase fire safety in hospitals'. However, the exact statement of the 'problem' might alter as the study progressed. For example, it might improve fire safety to reduce the number of electrical appliances in a hospital, but then the absence of a particular electrical instrument might have a very damaging effect with respect to some other aspect of the system. There will be conflicting objectives. People with different points of view need to be brought in. Also it will usually be the case that limitations of current resources allocated, or to be allocated, to the study (together with similar limitations and factors, e.g. temporal) constitute part of the problem itself.

Considering the hospital fire safety problem further, some of the relevant sub-systems might be:

1. Patients.

2. Nursing and medical staff.

3. General public.

4. National Health Service.

5. Department of Health and Social Security.

6. Home office

7. 'Design system' for construction of hospitals.

8. 'Fire safety design system' for construction of hospitals.

9. Hospital 'fire safety system'. (After construction.)

10. Fire brigade.

11. Ambulance service.

12. Local authority.

13. Fire research system.

14. Technical systems.

15. Systems directly associated with the chemistry and physics of fire processes.

16. Manufacturers of fire safety and other equipment used in hospitals.

17. British Government.

18. British socio-economic system.

19. International socio-economic system.

Most of these sub-systems overlap. The order given above is not meant to imply any kind of 'order of importance'; it is simply a list of some of the systems which might be pertinent to a study. Other systems may also be relevant. 
(2) Development of Models with Respect to the Systems Involved. A model is a representation of an aspect of 'reality' and expresses, amongst other things, the point of view of the person constructing it. The construction of models will already have started in stage 1. There are many different types of model, ranging from broad verbal statements to deterministic mathematical models. One may also consider physical models. In addition to models which are intended to represent a situation in a general manner, there are also 'simulation' models which are based on the generation of specific cases. Mathematical probabilistic models of this type might use, for example, the Monte Carlo technique. An example of a physical simulation model would be the representation of the flow of a river and its tributaries by flow of electrical current through wires.

Most of the systems we encounter have 'objectives' associated with them. That is, there may be aims and expectations which people have with respect to a system. A person or a group of people may want one or more things from a system. However, the objectives of a person or group may conflict. Also, that which a person or group wants. from a system may conflict with that which another person or group wants from the system. For example, the objectives of a manufacturer of motor cars will be different from the objectives of a buyer of a motor car. In general, for any complex system, there will almost certainly be conflicts of objectives. Clarification and understanding of objectives and how they arise is necessary.

The structures, processes and forces existing within systems and crossing system boundaries need to be investigated. Flows of information and material both within systems and between systems need to be understood. There may also be constraints for a given system. For example, the amount of money received by a local authority from government may be fixed.

The models should help us to understand better the relationships within the system as a whole.

However, a word of warning is necessary regarding the use of models. All models have Iimitations and it is vital to be aware of what those limitations are. The assumptions, both explicit and implicit, in each model must be clearly realized. It is as important to know what a model cannot say as to know what it can say. This is true for both quantitative and qualitative models. In particular, for quantitative models, it is important not to attach an unjustified significance to numbers which result. At an obvious level. there are uncertainties which will be associated with models. For numerically quantitative models these may be expressed as, for example, 'ernors', 'confidence limits' etc. Numerical results should be seen in their context. According to the mathematician Gauss a lack of appreciation of the value of mathematics is "nowhere revealed so clearly as by meaningless precision in numerical studies".

Another issue raised in the application of specific methods is that models, and techniques in general, may on occasion be applied to situations for which they are not appropriate. It has been said that this is the most common form of mis-use (3).

In addition to these 'overt' points there may also be mis-construction at a deeper level. A simplistic approach can sometimes lead one to effectively ascribe to models powers which they do not possess. (One might almost call this a 'fetishism' which may be associated with models.) For example, if one wishes to compare risk situations then a relevant quantity to take into account would be the probability of fatality associated with each risk. It would, however, be foolish to pretend that a comparison of the probabilities 
of fatality would represent a comparison of the situations which may give rise to fatality. Such over-simpliicty is sometimes an implied assumption if not an explicit one. There are many different dimensions involved in each risk and not all of these dimensions may be quantifiable; mathematical models cannot provide a 'correct answer'. A comparison of the risk associated with cardio-vascular disease and that associated with road accidents is not straight forward. Looking at the probabilities of fatality is to consider only one aspect of these risk situations. In general, qualitatively different facets should not be collapsed onto a one-dimensional scale.

Further, it may well not be possible in practice to associate numbers with many of the characteristics involved even if it may be possible to do so in principle. If we effectively insist, say, that a hazard only really exists if plausible numbers can be associated with it we shall be limiting our conceptualization dramatically. Also, considering things in purely quantitative terms may lead to a false sense of security because if numbers cannot be attached to a characteristic then there may be a tendency to ignore it; conceptual features may be lost. More generally, we must realize that all explanation is interpretive and context-dependent and, as Thomas has pointed out (4), how we measure fire safety is itself a value-judgement.

Having sounded these cautionary notes it must be said that the appropriate use of models may be of great help in enabling us to comprehend things. Both deterministic and probabilistic models may aid us and one probabilistic technique is seen in the stochastic model which has been developed by the author. Many of the assumptions contained in that model are not firmly based and it needs to be improved. Clarification of the assumptions and limitations is contained in the references. The purpose of that technique is to afford an idea of the likely number of fatalities given that a fire starts in a hospital ward. An estimate may thereby be found for the changes in the likely number of fatalities which would be expected to result from changes in the ward sub-system. It is described further in the Appendix.

(3) Examination of Specific Past Failure Situations. Studies of past fires are obviously of crucial importance. Each past fire should itself be examined from an overall systemic point of view. 'Near miss' occasions should be studied i.e. occasions which very nearly could have produced a failure but in fact did not. ('Failure' might be taken to mean, for example, 'injury or death due to fire'.)

Information, both statistical and non-statistical, should be considered. In the case of hospitals information afforded by hospital staff could prove to be very useful.

(4) Select Areas for Further Research or Investigation. Both experimental and/or non-experimental work might be pursued. For example, it might be decided that it would be useful to carry out some specific tests. Different kinds of survey could be considered. In the hospital case it might be decided to ask staff to answer survey questions.

(5) Consideration of 'The Problem' in the Light of the Earlier Stages. Generation of Ideas on how to Tackle 'The Problem'. Radical changes in the systems may be necessary. Given the understanding of the situation reached so far, what possible changes seem suitable as a means of eliminating or reducing the 'problem'? Alternative ideas may be tentatively suggested.

(6) Evaluation of Possible Courses of Action and Consequences. Each of the alternatives to emerge in the previous stage should be considered in detail. 
An attempt must be made to elucidate the full implications of each alternative.

\section{(7) Carrying Out Action}

(8) Appraisal. Whatever actions are carried out the study is never 'finished'. It will always be necessary to re-think old ideas.

Before leaving this discussion of the stages of the methodology it is important to make the general point that the investigators themselves are also part of the system under study and this must be realized (5).

\section{CONCLUSION}

Fire safety needs to be examined from a systemic point of view and not in a disjointed or narrow fashion. Fire is a product of a system and it is necessary to understand the 'dynamic wholeness' of things in order to understand the failure.

To carry out a study from a systemic point of view it is necessary to have a guiding structure (or methodology) which is dynamic and not static. Within a methodology specific techniques may be employed which have specific tasks. It must be emphasised, however, that a particular technique would have a relatively limited remit and would only consitute a part of a systemic approach. A distinction must be drawn between a theoretical model which attempts to acount for different factors in trying to answer a fairly narrow question and a systemic consideration of the problem as a whole. A possible outline for such a methodology has been suggested and it is hoped that such a framework would help in the task of exploring as many aspects of the problem as possible.

Essentially, the central theme of this paper has been the need to consider things in their entirety. Concern about fire safety has been the 'starting point' for this consideration. However, as has been mentioned, in any complex situation involving human beings there will be conflicting objectives and in any action it is necessary to attempt to deal with all of these objectives. We need to try to develop a system within which conflicts and contradictions do not arise, as far as we possibly can.

Fire safety has been the starting point in this paper but if we view things as an entirety then in principle all aspects of a system should enter into consideration no matter what is the starting point.

In short, one can rarely, if ever, make a straight forward 'fire safety decision'. Generally, one can only make a 'decision' which has 'fire safety consequences', amongst others. Usually a decision will have numerous ramifications, implications and facets. Many people, bringing in different points of view, need to be involved in the process.

To close, a line from the New England poet Enily Dickinson puts things rather well:

"In broken mathematics we estimate our prize,

Vast in its fading ratio to our penurious eyes."

We need to be able to develop eyes which are no longer penurious but are capable of seeing the nature, complexity and inter-relationship of the world we live within. In such a context mathematical models may have a truly valuable contribution to make. 
This Appendix concentrates on a brief description of a type of mathematical model which may be used as a part of a guiding methodology. It serves as an example of a general class of techniques but it is not meant to be implied that this kind of technique is necessarily 'better' than other models. As stressed in the main text, many different kinds of model should be considered and all techniques should be employed with caution and circumspection.

This Appendix does not describe an application of the methodology suggested. It attempts to answer a specific question and considers different causal factors as parts of the model. Such a technique would form only a small part of a systemic approach.

Before describing the stochastic model reference may be made to three logic-trees which have been constructed for three past fire disasters. Each tree was used to assess the reduction in the likelihood of the occurrence of the disaster if things had been different. The three fires considered were the Coldharbour Hospital fire (6), the Fairfield Home fire (7) and the St. Crispin Hospital fire (8).

In addition to these specific logic-trees a stochastic model has been developed which is intended to give an idea of the likely number of deaths resulting from a fire in a given space. The development of the fire in terms of heat, smoke and toxic gases is considered as well as the response of those present and evacuation characteristics. The model has been constructed for hospital wards and has been applied to the ignition of a bed having a polyurethane mattress. Two types of ward have been looked at, the first being of 'Nightingale' design containing thirty, bed-bound, non-ambulant patients and the second being a ward consisting of six-bedded bays containing similar patients $(9,10)$. Further considerations, including sensitivity studies, have also been carried out (11).

A stochastic framework has been devised by considering a number of 'critical events' which the fire may pass through and the times between critical events. Particular rates of heat release are associated with the critical events and these are given in table 1. The probability of a fire going through a critical event given that it has gone through an earlier one is represented by a 'transition probability', $P_{i}$. Probability density functions have been assumed for the times between crltical events and fires have been simulated using the Monte Carlo method. Each fire passes through one of six chains with number 1 corresponding to the most trivial fire and number 6 the most serious. Table 2 shows the relative frequency of each chain and the numbers of fires corresponding to given numbers of fatalities for each chain. This is for the Nightingale ward calculation.

For the bay calculation it was found that the likelihood of multiple fatalities resulting is higher than for the Nightingale arrangement but that the mean number of fatalities for 500 simulations is approximately half that for the Nightingale ward.

Sensitivity studies have been conducted for the Nightingale calculation by looking at the effects of changes in the assumptions on the mean number of deaths. The most striking change is seen upon alteration of each of the first two transition probabilities, all else remaining the same, (Fig. 2). It has also been found that the calculated mean number of fatalities is approximately halved if the smoke and gases are assumed to stratify rather than fill the ward uniformly. 
TABLE 1 - Critical Heat Event (CHE) Definitions.

(CE1 refers to the first critical event).

$\begin{array}{ll}\text { CE1 } & \text { Ignition } \\ \text { CHE2U } & \text { Fire passes through } 2 \mathrm{kw} \text { on way up } \\ \text { CHE3U } & \text { Fire passes through } 50 \mathrm{kw} \text { on way up } \\ \text { CHE4U } & \text { Fire passes through } 1000 \mathrm{kw} \text { on way up } \\ \text { CHE5U } & \text { Fire passes through } 10,000 \mathrm{kw} \text { on way up } \\ \text { CHE6U } & \text { Fire passes through a level } \dot{\mathrm{q}}_{\mathrm{m}}^{\text {(upwards) }}\end{array}$

TABLE 2 - The relative frequency of each chain and the numbers of fires corresponding to given numbers of fatalities for each chain.

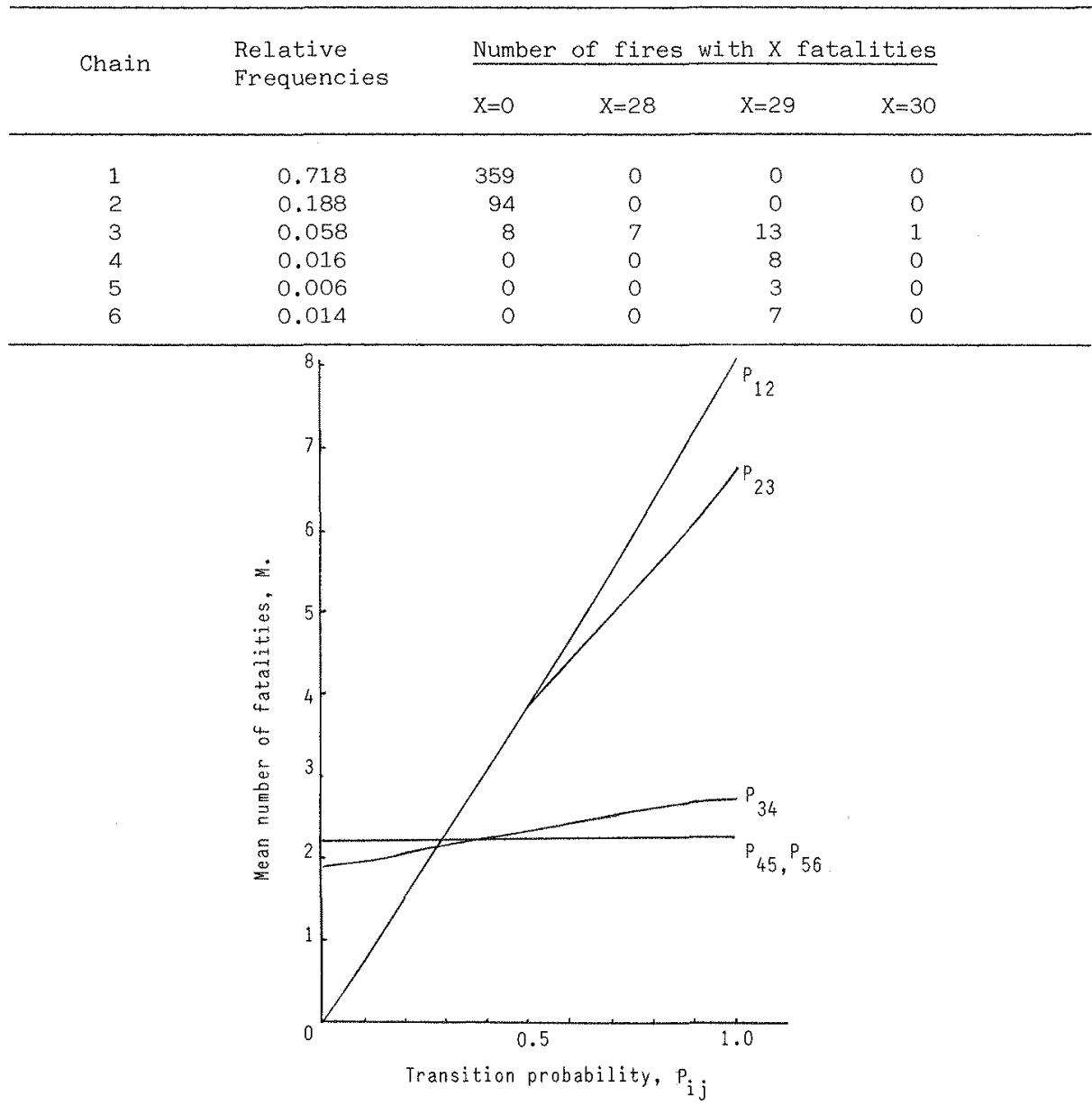

FIGURE 2 - Variation in mean number of fatalities, M, with each transition probability, $P_{i j}$.

(Uniess otherwise: $P_{12}=P_{23}=0.3 ; P_{34}=0.4 ; P_{45}=0.5 ; P_{56}=0.6$ ) 


\section{REFERENCES}

1. Beer, S., 'Below the Twilight Arch-A Mythology of Systems'; In Proceedings of the First Systems Symposium, Case Institute of Technology, 1961.

2. M'Pherson, P.K., 'A Perspective on Systems Science \& Systems Philosophy' Futures, Vol. 6, no. 3, pp 219-239, June 1974.

3. 'Systems Performance-Human Factors and System Failures', The Open University Press.

4. Thomas, P.H., 'Fire Safety: Some General Aspects of Research, Regulation and Design'; In conference entitled "Systems Approach to Fire Safety in Buildings", Tsukuba, Japan, 29-30 August, 1979.

5. Boulding, K.E., 'General Systems as a Point of View'; In proceedings of the Second Systems Symposium, Case Institute of Technology, 1964.

6. Beard, A.N., 'Applying Fault-Tree Analysis to the Coldharbour Hospital Fire', "Fire", vol. 71, pp 517-519, 1979.

7. Beard, A.N., 'A Logic-Tree Approach to the Fairfield Home Fire"; "Fire Technology", vol. 17, no. 1, pp 25-38, February 1981.

8. Beard, A.N., 'A Logic-Tree Approach to the St. Crispin Hospital Fire', "Fire Technology", vol. 19, no. 2, pp 90-102, May 1983.

9. Beard, A.N., 'A Stochastic Model for the Number of Deaths in a Fire'; "Fire Safety Journal", vol. 4, pp 169-184, 1981/82.

10. Beard, A.N., 'A Stochastic Model for the Number of Deaths Resulting from a Fire in a Bay in a Hospital Ward'; "Fire Safety Journal", vol. 6, pp $121-128,1983$.

11. Beard, A.N., 'A Stochastic Model for the Number of Deaths in a Fire: Further Considerations", "Fire Safety Journal", vol. 8, pp 201-226, 1985.

\section{ACKNOWLEDGEMENTS}

I would like to thank Professor David Rasbash for his comments on this work. Also, I am grateful to the Fire protection Association, London, for permission to use material from the article "Towards a Rational Approach to Fire Safety" which was published in 'Fire Prevention Science and Technology', No. 22, pp 16-20, 1979. This work was supported financially by the Science \& Engineering Research Council and the Department of Health \& Social Security. 